\title{
A new mathematical model for fitting an HPL radioimmunoassay curve
}

\author{
B. R. HARDING, RITCHIE THOMSON, AND A. R. CURTIS
}

From the Radiochemical Centre, Amersham, Bucks., and the

Theoretical Physics Division, AERE, Harwell, Berks, England

SYNOPSIS A number of mathematical models have been tested for their suitability in representing the dose response curve of a specific assay of the hormone human placental lactogen (HPL). ${ }^{1}$

A new equation $\mathrm{Y}=\mathrm{B}[0 \cdot 14+1 /[1+\mathrm{C} \log [1+\exp [\mathrm{X}-\mathrm{D}]]]$ where $\mathrm{Y}$ is the percentage activity or counts bound to the antibody and $\mathrm{X}$ is the HPL concentration is proposed as representing the overall shape of the curve. This model is shown to give both an accurate representation of the curve and to allow reproducible determination of an unknown over a number of occasions. A number of other models are compared. The new model allows automatic calculation of HPL concentrations from a standard curve using a computer.

One important current development in the routine implementation of radioimmunoassay in clinical chemistry is the use of automatic data processing. Modern large computers reduce the amount of manpower required in calculation and can give more objective, reliable, and rapid results than manual methods. The use of numerical calculation depends on some kind of model which represents the doseresponse curve of the assay under consideration. This paper considers various possible models to represent the dose-response curve for one specific assay of HPL. An example of the curve is shown in the figure.

In the design of this assay kit ${ }^{2}$, working concentrations of antiseium and labelled HPL were set at much higher levels than would normally be encountered in the radioimmunoassay of many hormones. This allowed the direct assay of the relatively high concentrations of HPL encountered in the second half of pregnancy (Chard, 1973). Greater precision in the range 3-6 $\mu \mathrm{g} \mathrm{HPL} / \mathrm{ml}$ was achieved by maximizing the slope of the dose response line in this region. A consequence of this design is that the slope of the curve in the $0-1 \mu \mathrm{g} \mathrm{HPL} / \mathrm{ml}$ region approaches zero as the antibody is almost unsaturated here.

A restriction is set by the number of standard points available. Serum standards at four concentrations are supplied by the manufacturer. These

\footnotetext{
${ }^{1}$ The hormone human placental lactogen (HPL) is also known as human chorionic somatomammotrophin (HCS).

'The HPL immunoassay kit produced by the Radiochemical Centre, Amersham, England.

Received for publication 31 August 1973.
}

four have proved satisfactory in defining the curve for manual interpolation where the mean of duplicate determination at each point is used. A similar restriction was set for any automatic method.

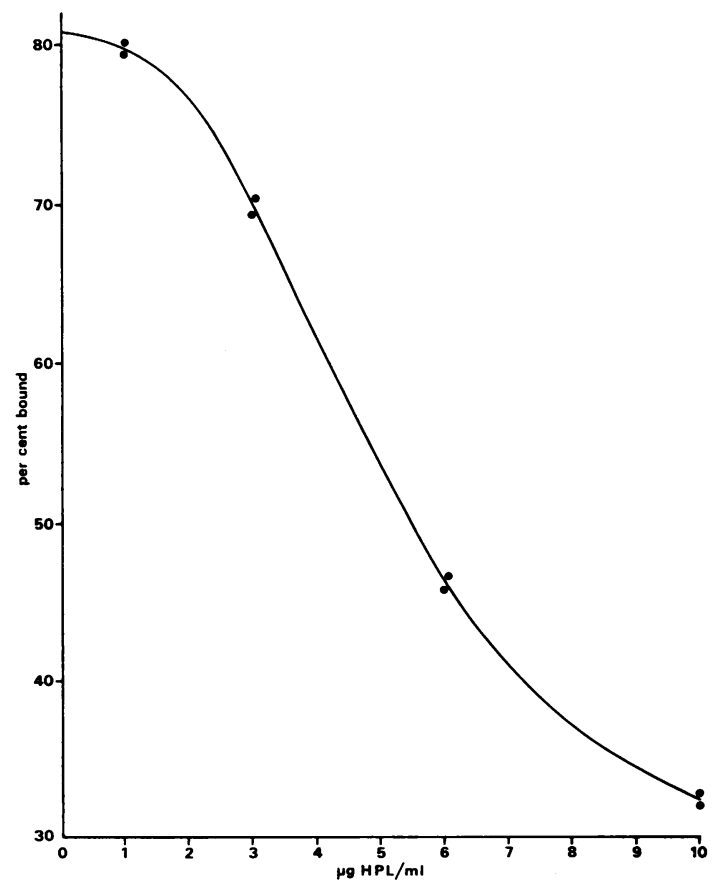

Fig Human placental lactogen dose response curve. 


\section{Models for Curve Fitting}

A number of different models have been proposed for representing radioimmunoassay dose response curves. The most generally used model is the loglogit curve which has been discussed by a number of authors (Rodbard and Lewald, 1970; Täljedal and Wold, 1970; Healy, 1972). This curve gives a good fit in many assays but has not been found suitable to represent the HPL assay curve in this case where the slope approaches zero at zero HPL concentration.

There are a limited number of suitable functions which may possibly be used to represent a full immunoassay curve as shown in the figure. One suitable function has been suggested by Burger, Lea, and Rennie (1973). The fit in the case of HPL was rather poor but this served as a useful comparison with other possible functions and is hence included in the discussion.

Another possible function was an inverted third order polynomial. Polynomials can give excellent representations of many curves but are open to criticism for a number of reasons. Normally a reasonable number of points must be used to define a polynomial. The use of a third order polynomial, which must automatically pass through the four data points provided (at HPL concentrations of 1 , 3,6 , and $10 \mu \mathrm{g} / \mathrm{ml}$ ) is suspect because the curve is not controlled in any way to have the correct shape of an immunoassay curve. However, polynomials are very simple functions to calculate on a programmable calculator and the model could therefore be suitable for laboratories where a more sophisticated technique cannot be used.

Two new curves are proposed. Both functions are constrained to have the 'correct' sliape for the HPL dose response curve. The Amersham-4 curve has four parameters to be fitted and thus will pass exactly through each data point. This curve has the general properties that it is almost flat near the origin and then turns over to descend steeply. The shape of the curve tends towards a rectangular hyperbola reaching to a constant non-zero value at high HPL concentration.
B. R. Harding, Ritchie Thomson, and A. R. Curtis $\frac{\varrho}{\bar{\Xi}}$

The Amersham-3 curve was derived from the Amersham -4 curve by noting that in practice the 을 ratio $\mathbf{A} / \mathbf{B}$ was approximately constant. This ratio $\vec{\Rightarrow}$ is held constant at $0 \cdot 14$ in the Amersham-3 curve. $\stackrel{?}{+}$ This builds in knowledge about the shape of the curve and allows a limited degree of data smoothing to be applied. The penalty paid is a more rigidly fixed curve with one less parameter to fit.

To decide the best model a comparison of the curves over a number of data sets was carried out as $\vec{\circ}$ has already been done, for example, for an insulin assay (Meinert and McHugh, 1968). Computer $\vec{\omega}$ programs were written to calculate parameters in 5 each case. These programs minimize the mean square ? discrepancy of counts on percentage bound from the line, where four parameters are available this discrepancy is zero. No weighting of the data points ? was used.

\section{Criteria for Assessment of Curve Suitability}

The criteria used in determination of the curve quality were that, using the four standard points provided with duplicate determinations at each point, the curve derived should represent the true dose response curve accurately and reproduciblp One basis for comparison might be with the per formance of experienced operator(s). We expeg. these criteria to apply over the range of typical assa curves.

A number of assay curves were derived over different ages of labelled HPL, antiserum, and operators to represent typical curves. Also included were two 'worst possible cases' using abnormal reagentsa diluted antiserum and a preparation of labelled HPL used well beyond its expiry date. In each case the four standards were accurately mixed to provide intermediate standards. The comparison between true and calculated values in these intermediate points allowed the accuracy of the models to be assessed.

A further set of data was available, in which three unknown control sera were run a number of times. This data was reprocessed through each program

\begin{tabular}{lrl}
\hline Manual & Curve drawn by experienced operator(s) using Flexi Curve \\
Burger et al (1973) & $\mathbf{y}=\frac{\mathbf{A}}{\mathbf{B}+\mathbf{X}^{\mathbf{c}}}$ \\
Third order inverted polynomial & $\mathbf{x}^{2}=\mathbf{A}+\mathbf{B y}+\mathbf{C} \mathbf{y}^{2}+\mathbf{D} \mathbf{y}^{3}$ \\
Amersham-4 & $\mathbf{y}=\mathbf{A}+\mathbf{B} /[1+\mathbf{C} \log [1+\exp [\mathbf{x}-\mathbf{D}]]]$ \\
Amersham-3 & $\mathbf{y}=\mathbf{B}[0 \cdot 14+1 /[1+\mathbf{C} \log [1+\exp [\mathbf{x}-\mathbf{D}]]]$ \\
\hline
\end{tabular}

Table I Curves tested

$\left.{ }^{2} y=\begin{array}{c}\% \text { activity bound to antibody } \\ \text { or counts bound to antibody }\end{array}\right\}$ uncorrected for blank

${ }^{2} \mathrm{x}=$ HPL concentration $(\mu \mathrm{g}$ HPL/ml) 


\begin{tabular}{|c|c|c|c|c|c|c|c|c|c|c|c|c|c|c|}
\hline Model & Mean & $S E$ & Mean & $S E$ & Mean & $S E$ & Mean & $S E$ & Mean & $S E$ & Mean & $S E$ & Mean & $S E$ \\
\hline True value & 0 & - & 2 & - & 4 & - & 5 & - & 7 & - & 8 & - & 9 & - \\
\hline Manual & 0.56 & 0.08 & $1 \cdot 71$ & 0.08 & $4 \cdot 01$ & 0.06 & 5.06 & 0.06 & 7.02 & 0.08 & 8.01 & 0.08 & 9.07 & $0 \cdot 11$ \\
\hline Burger et al (1973) & 0.73 & 0.06 & 1.68 & 0.05 & $3 \cdot 99$ & $0 \cdot 10$ & $5 \cdot 18$ & 0.05 & $7 \cdot 31$ & 0.07 & $8 \cdot 16$ & 0.04 & $8 \cdot 99$ & 0.06 \\
\hline $\begin{array}{l}\text { Third order inverted } \\
\text { polynomial }\end{array}$ & $0 \cdot 57$ & 0.09 & $1 \cdot 80$ & 0.08 & $3 \cdot 93$ & 0.04 & $4 \cdot 98$ & 0.07 & $7 \cdot 09$ & 0.08 & $8 \cdot 07$ & 0.07 & $9 \cdot 03$ & $0 \cdot 14$ \\
\hline Amersham-3 & $0 \cdot 20$ & 0.11 & $1 \cdot 84$ & 0.08 & $3 \cdot 91$ & 0.04 & $4 \cdot 96$ & 0.04 & $7 \cdot 00$ & 0.07 & $8 \cdot 00$ & 0.07 & $9 \cdot 03$ & 0.09 \\
\hline Amersham-4 & 0.24 & $0 \cdot 11$ & $1 \cdot 86$ & 0.05 & $3 \cdot 92$ & 0.03 & 4.97 & 0.05 & $7 \cdot 00$ & 0.08 & $7 \cdot 96$ & 0.06 & 9.09 & 0.11 \\
\hline
\end{tabular}

Table II Comparsion of models for accuracy in representing curve ${ }^{1}$

${ }^{1}$ All results in $\mu \mathrm{g} \mathrm{HPL} / \mathrm{ml}$ serum.

$\mathrm{SE}=$ standard error, ie, standard deviation of the mean

and the mean standard deviation of each unknown was calculated. Since a larger quantity of data was available in this case (18 assays) it was possible to derive more accurate statistical comparisons of the reproducibility of the curves. For the manual fit in this case the curves were drawn by a number of operators (nine in all).

\section{Results and Discussion}

The calculated mean and standard errors of the intermediate standards are shown in table II. A comparison between the true and calculated values shows in general excellent agreement for most curves except at zero and $2 \mu \mathrm{g}$ HPL. The exception is the Burger curve which shows significant deviations between 5 and $7 \mu \mathrm{g}$ HPL. This is an important region of the curve and because of such systematic deviations, this curve is not acceptable.

In the $0-2 \mu \mathrm{g}$ HPL region the precision of the assay is low and this area is relatively unimportant; it is worth noting that the Amersham curves give the best representation in this region. However, any of the curves except the Burger fit would be acceptable as giving a fit of suitable precision. Indeed, the excellence of the manual fit, where all the curves were drawn by one experienced operator, is remarkable.

Table III summarizes the results obtained over 18 assays with three control sera. With the low control
(A) the standard deviations are comparable with all the models while with the high control (C) the manually drawn curves are significantly worse than the best models in this region. The middle standard (B) lies in the critical 3-6 $\mu \mathrm{g} \mathrm{HPL} / \mathrm{ml}$ region where the highest precision is required. Here the Burger and Amersham-3 curves are best on this criterion but the Burger curve has already been rejected for systematic deviations. It is clear that the Amersham-3 curve gives significantly better reproducibility than the manual or inverted third order polynomial methods. The Amersham-3 curve is also appreciably better than the Amersham- 4 curve but the statistical significance is not high.

The Amersham-3 curve is thus the most suitable of all the models examined. It gives both an accurate representation of the HPL curve over a range of practical data and a more reproducible result on a control in the region of highest precision.

There are two alternatives to this. The Amersham-4 curve gives no data smoothing and a higher standard deviation in the critical range. However, with four parameters to fit it might give a more accurate representation of curves of abnormal shape. It is very doubtful that this would arise in practice. The inverted third order polynomial gives a good representation of the curve but a significantly worse standard deviation on the control serum in the critical area. We would not recommend its use in general. However it has the advantage of simplicity of calculation

\begin{tabular}{|c|c|c|c|c|c|c|}
\hline \multirow[t]{3}{*}{ Model } & \multicolumn{6}{|c|}{ Control } \\
\hline & \multicolumn{2}{|l|}{$A$} & \multicolumn{2}{|l|}{$\boldsymbol{B}$} & \multicolumn{2}{|l|}{$C$} \\
\hline & Mean & $S D$ & Mean & $S D$ & Mean & $S D$ \\
\hline $\begin{array}{l}\text { Manual } \\
\text { Burger et al (1973) } \\
\text { Third order inverted polynomial } \\
\text { Amersham-3 } \\
\text { Amersham-4 }\end{array}$ & $\begin{array}{l}1 \cdot 78 \\
1 \cdot 68 \\
1 \cdot 84 \\
1 \cdot 86 \\
1 \cdot 88\end{array}$ & $\begin{array}{l}0 \cdot 248 \\
0 \cdot 240 \\
0 \cdot 237 \\
0 \cdot 244 \\
0 \cdot 254\end{array}$ & $\begin{array}{l}4 \cdot 87 \\
4 \cdot 98 \\
4 \cdot 73 \\
4 \cdot 84 \\
4 \cdot 78\end{array}$ & $\begin{array}{l}0 \cdot 195 \\
0 \cdot 145 \\
0 \cdot 258 \\
0 \cdot 124 \\
0 \cdot 177\end{array}$ & $\begin{array}{l}7 \cdot 45 \\
7 \cdot 85 \\
7 \cdot 59 \\
7 \cdot 57 \\
7 \cdot 52\end{array}$ & $\begin{array}{l}0 \cdot 356 \\
0 \cdot 201 \\
0 \cdot 256 \\
0 \cdot 203 \\
0 \cdot 260\end{array}$ \\
\hline
\end{tabular}

Table III Comparison of models for reproducibility of controls

'All results in $\mu \mathrm{g} \mathrm{HPL} / \mathrm{ml}$ serum

$\mathrm{SD}=$ Standard deviation 
and a laboratory which has only a programmable calculator might choose to use it provided they accept its limitations.

\section{Computing Consideration}

The Amersham-3 and -4 curves are both highly nonlinear in their parameters. Sophisticated programs have been prepared for fitting both curves using ICL Fortran and subroutines from the Harwell library (Hopper, 1971). A simplified program for the Amersham-3 curve has been prepared in Basic using the techniques presented by Burger et al (1973). No problems of convergence have been seen in about 100 sets of data using the Basic program.

\section{Conclusion}

The Amersham-3 curve has been shown to give accurate and reproducible answers for a specific assay of HPL. It has been designed for this assay and cannot be used for other assays in the form presented. The general curve shape may prove extremely useful in representing other assay curves and it is proposed to proceed to investigate this possibility.
The advantage of the Amersham curve type is that $\frac{0}{5}$ it may give a more accurate representation of the curve near zero dose and allow a more precise $\overrightarrow{\vec{F}}$ determination of assay sensitivity.

We wish to express our gratitude to Mr J. Bryant $\frac{}{\bar{N}}$ who prepared the Basic version of the Amersham- $3 \overrightarrow{\mathrm{D}}$ fit and to Dr D. Brunwin who provided some of the test data.

\section{References}

Burger, H. G., Lee, V. W. K., and Rennie, G. C. (1973). A generalised $\vec{\omega}$ computer program for the treatment of data from competitive protein-binding assays including radioimmunoassays. J. Lab.융 clin. Med., 80, 302-312.

Chard, T. (1973). HPL levels as a guide to foetal well-being during $\mathcal{O}$ pregnancy. Medical Monograph 8. Radiochemical Centre, $\vec{N}$ Amersham.

Healy, M. J. R. (1972). Statistical analysis of radioimmunoassay 6 data. Biochem. J., 130, 217-210.

Hopper, M. J. (1971). Harwell subroutine library. HMSO-HL 71/4679 (CB).

Meinert, C. L., and McHugh, R. B. (1968). The biometry of an isotope displacement immunologic microassay. Math. Biosci., 2, 319-338.

Rodbard, D., and Lewald, J. E. (1970). Computer analysis of radio ligand assay and radioimmunoassay data. In Steroid Assay by Protein Binding (Karolinska Symposium on Research methods in Reproductive Endocrinology, 2; Acta Endocrinologica Suppl. 147) edited by E. Diczafalusy, pp. 79-103.

Täljedal, I. B., and Wold, S. (1970). Fit of some analytical functions $\overrightarrow{0}$ to insulin radio-immunoassay standard curves. Biochem. 119, 139-143. 